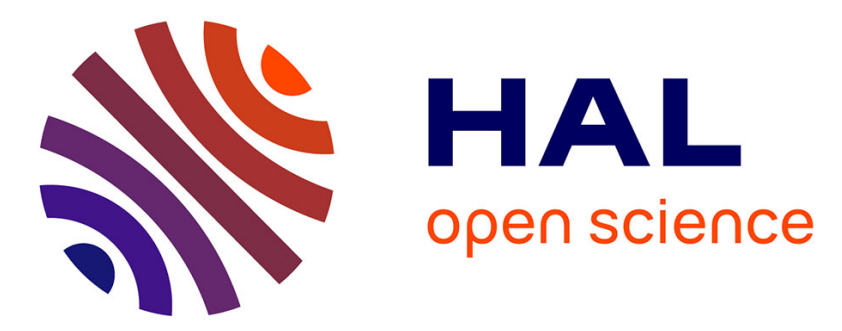

\title{
Les complexes volcaniques Rhyolithiques quaternaires d'Anatolie centrale (Göllü Dag et Acigöl, Turquie) : Genèse, instabilité, contraintes environnementales
}

Damase Mouralis, Jean-François Pastre, Catherine Kuzucuoğlu, Ahmet Türkecan, Yelda Atici, Ludovic Slimak, Hervé Guillou, Stéphane Kunesch

\section{To cite this version:}

Damase Mouralis, Jean-François Pastre, Catherine Kuzucuoğlu, Ahmet Türkecan, Yelda Atici, et al.. Les complexes volcaniques Rhyolithiques quaternaires d'Anatolie centrale (Göllü Dag et Acigöl, Turquie): Genèse, instabilité, contraintes environnementales. Quaternaire, 2002, 13 (3), pp.219-228. 10.3406/quate.2002.1714. hal-02478048

\section{HAL Id: hal-02478048 \\ https://hal.science/hal-02478048}

Submitted on 19 Oct 2020

HAL is a multi-disciplinary open access archive for the deposit and dissemination of scientific research documents, whether they are published or not. The documents may come from teaching and research institutions in France or abroad, or from public or private research centers.
L'archive ouverte pluridisciplinaire HAL, est destinée au dépôt et à la diffusion de documents scientifiques de niveau recherche, publiés ou non, émanant des établissements d'enseignement et de recherche français ou étrangers, des laboratoires publics ou privés. 


\title{
LES COMPLEXES VOLCANIQUES RHYOLITIQUES QUATERNAIRES D'ANATOLIE CENTRALE (GÖLLÜ DAG ET ACIGÖL, TURQUIE) : GENĖSE, INSTABILITÉ, CONTRAINTES ENVIRONNEMENTALES
}

\author{
Damase MOURALIS ${ }^{1}$, Jean-François PASTRE', Catherine KUZUCUOGLU ${ }^{1,2}$, \\ Ahmet TÜRKECAN ${ }^{3}$, Yelda ATICI ${ }^{3}$, Ludovic SLIMAK ${ }^{4}$, Hervé GUILLOU ${ }^{5}$, \\ et Stéphane KUNESCH'.
}

\begin{abstract}
RÉSUMÉ
Le volcanisme calco-alcalin d'Anatolie centrale est à l'origine de changements rapides et conséquents des environnements quaternaires. Les deux grands complexes rhyolitiques du Göllü Dag et d'Acıgöl intègrent des caldeiras, de nombreuses pyroclastites et des extrusions laviques s'intercalant avec des formations détritiques variées. Le premier a fonctionné du Pléistocène inférieur jusqu'au Pléistocène moyen. Le second s'étend de la fin du Pléistocène moyen au Pléistocène supérieur.

Les données présentées permettent de suivre l'évolution rapide de ces complexes, de définir la caldeira du Göllü Dag et de redéfinir celle d'Acigöl. Chacun de ces complexes à fonctionné en trois phases, pré-, syn- et post-caldeira. Ces phases sont responsables de l'éruption de volumes très différents de magma et de modifications d'ampleur variable des environnements. Les phases syn-caldeira sont responsables des changements les plus importants et les plus rapides. Elles se traduisent par la mise en place de grands volumes de pyroclastites et une modification fondamentale des reliefs et du réseau hydrographique préexistant. Les phases post-caldeira induisent des changements plus modestes, marqués par l'extrusion répétée de dômes dont nous décrivons un modèle de mise en place original associant phréatomagmatisme, intrusion de dykes annulaires d'obsidienne, activité peléenne, et extrusion rhyolitique. Ces dykes d'obsidienne sont à l'origine d'une importante fréquentation de la région par les sociétés humaines au cours du Paléolithique et du Néolithique. La moindre ampleur des volumes émis et l'étalement des éruptions dans le temps permet l'interstratification des pyroclastites avec des formations alluviales et colluviales pléistocènes. Cette évolution définit des séquences variées où s'intercalent des téphra-repères et des industries paléolithiques. Leur étude permet d'analyser les différents facteurs contrôlant leur mise en place et de les resituer dans un cadre téphrostratigraphique largement inédit.
\end{abstract}

Mots-clés : Turquie, Anatolie Centrale, Cappadoce, Volcanisme calco-alcalin, Complexes rhyolitiques, Téphra, Obsidienne, Paléolithique, Environnements quaternaires

\section{ABSTRACT}

QUATERNARY RHYOLITIC VOLCANIC COMPLEXES FROM CENTRAL ANATOLIAN (GÖLLÜ DAG AND ACIGÖL) : GENESIS, INSTABILITY AND ENVIRONMENTAL CONSTRAINTS.

During the Quaternary, important and rapid environmental changes occurred in central Anatolian in relation with calco-alcaline volcanism. This volcanic activity generated two large rhyolitic complexes integrating calderas, several pyroclastic deposits and lavic extrusions interstratified with various detritic formations. Activity of the Göllï Dag and the Acıgöl complexes lasted respectively from the lower Pleistocene until the middle Pleistocene, and from the middle Pleistocene until the upper Pleistocene.

Results presented here allow i) to evidence the Göllü Dag caldera for the first time, ii) to define the extension of the Acıgöl caldera according to new data, and iii) to reconstruct the successive phases in the evolution of both complexes. These complexes present three main types of activity : pre-, syn-, and post-caldera, each timing being responsible for various environmental changes related to different pyroclastite deposition characteristics (volume, types, dynamics, ...). Syn-caldera activity induced the most important environmental changes, which affected palaeogeography and palaeohydrology. During post-caldera activity, changes are less important and concern the extrusion of local rhyolitic domes, in the frame of an original model of extrusion presenting phreatomagmatism associated with annular

\footnotetext{
I Laboratoire de Géographie Physique. UMR 8591, CNRS Université Paris 1, - 1 place Aristide Briand, 92195 Meudon (France). mouralis@cnrs-bellevue.fr 2 Institut français d’études anatoliennes, (IFEA), MAE, Nuru Ziya Sok. 22. PK 54. Beyoglu, 80072, Istanbul (Turquie).

3 Maden Tetkik ve Arama (MTA), Genel Mudurlugu, Jeoloji Etütleri Dairesi. Eskisehir Yolu. 06520 Ankara (Turquie)

4 ESEP. UMR 6636, Maison Méditerranéenne des Sciences de l'Homme, 5 rue du Château de l'Horloge, BP 647, 13094 Aix-en-Provence Cedex 2 (France).

5 Laboratoire des Sciences du Climat et de l'Environnement, (LSCE), CEA-CNRS, Avenue de la Terrasse. 91198 Gif-sur-Yvette (France).
} 
obsidian dykes, pelean eruptions and rhyolitic extrusion. During Palaeolithic and Neolithic times, mining and chopping of obsidian caused the noticeable presence and activity of human societies. In comparison with syn-caldera activities, the volumes of pyroclastites deposited during the post-caldera episodes were reduced, so that they are interbedded within alluvial and colluvial Pleistocene formations containing also some obsidian artefacts. This context offers interesting sequences where tephra layers, paleosol, reworked formations and archaeological (Palaeolithic) industries, give opportunities to set a quite precise chronology and to identify palaeogeographical changes. This study allows also to analyse factors controlling environmental changes in a volcanic region and to correlate these changes using a tephrostratigraphical framework detailed here for the first time.

Key-words : Turkey, Central Anatolian, Cappaducia, Calco-alcalin volcanism, Rhyolitic complexes, Tephra, Obsidian, Palaeolithic, Quaternary environments.

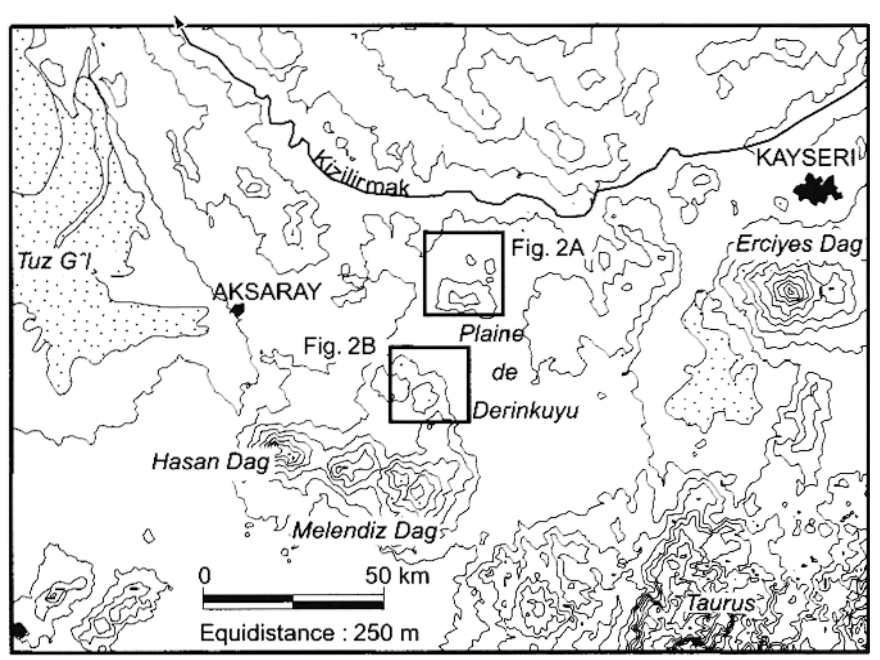

Fig. 1 : Localisation de la région étudiée

Fig. 1 : Location of the studied area.

\section{INTRODUCTION}

Les complexes rhyolitiques du Göllü Dag et d'Acıgöl sont localisés en Cappadoce (Fig. 1), dans la province volcanique d'Anatolie centrale qui a connu une importante activité volcanique depuis le Néogène. Cette activité est caractérisée par la mise en place de grandes nappes ignimbritiques (Le Pennec 1994) et par l'édification de strato-volcans andésitiques qui déterminent le cadre géomorphologique régional. Au Quaternaire, le volcanisme se manifeste par trois types d'activité. Deux grands strato-volcans, l'Erciyes Dag (Türkecan et al., 1998) et le Hasan Dag (Aydar \& Gourgaud, 1998) ont été actifs au cours du Pléistocène et probablement jusqu'à l'Holocène, comme semblent l'indiquer des témoignages historiques (Türkecan et al., 1998) et préhistoriques (Melaart, 1953). Cependant les produits de ces éruptions les plus récentes n'ont pas été retrouvés. Dans le même temps, un volcanisme peu différencié (basaltique) est responsable de l'édification d'appareils monogéniques : maars et cônes stromboliens, (Ercan, Yildirim \& Akbasli, 1987, Ercan, et al., 1991, Keller, 1974). Une activité rhyolitique est enfin responsable du fonctionnement des deux complexes du Göllü Dag et d'Acıgöl.

Les affleurements les plus anciens de la régions correspondent à des calcaires marmoréens paléozoïques à

\begin{tabular}{|llll|}
\hline Localisation & Age TF (Ma) & $\left(^{*}\right)$ & Réf. \\
\hline Complexe Du Göllü Dag & & & \\
Göllü Dag - Kayırll est & $1.480+/-0.09$ & A & $(1)$ \\
Göllü Dag - Kömürcü & $1.220+/-0.08$ & B & $(2)$ \\
Göllü Dag - Bozköy & $1.150+/-0.07$ & C & $(2)$ \\
Göllü Dag - Sirça Deresi & $0.980+/-0.06$ & D & $(2)$ \\
\hline Complexe d'Acıgöl : & & & \\
Acıgöl - Catköy & $0.200+/-0.04$ & E & $(1)$ \\
Bogazköy & $0.182+/-0.02$ & F & $(2)$ \\
Kocadag & $0.077+/-0.01$ & G & $(2)$ \\
Güneydag & $0.020+/-0.01$ & H & $(2)$ \\
Korudag & $0.020+/-0.01$ & I & $(2)$ \\
Maar d'Acıg̈l & $0.019+/-0.01$ & J & $(2)$ \\
\hline
\end{tabular}

Références : (1) Biggazi et al., 1997 ; (2) Bigazzi et al., 1993.

(*) Localisation sur les fig. 2A et B. - Location in fig. $2 A$ and $2 B$.

Tab. 1 : Âges (TF) des obsidiennes post-caldeira des complexes du Göllü Dag et d'Acigöl

Tab. : Post-caldeira obsidian fission-track ages from Göllï Dag and Actgöl complexes.

mésozoïques ainsi qu'à des granites et des roches ultrabasiques crétacés de nature ophiolitiques affleurant à l'ouest du complexe d'Acigöl (Atabey, 1989). Ensuite viennent les roches volcaniques néogènes : des ignimbrites (faciès Kızılkaya) arment les principaux reliefs au nord du complexe d'Acıgöl, alors qu'au sud, le massif de l'Erdas Dag témoigne de l'ancienne extension des strato-volcans andésitiques néogènes. Ces mêmes andésites arment le Sahinkalesi Tepe et le Melendiz Dag, qui limitent le complexe du Göllü Dag respectivement à l'ouest et à l'est. On observe ainsi que les roches les plus anciennes n'affleurent qu'aux limites des deux structures, mais jamais en leur centre.

Les deux complexes associent une caldeira dont l'effondrement est lié à la mise en place des principales pyroclastites, et des dômes extrudés postérieurement au sein de la structure d'effondrement (Fig. 2A et 2B). Les deux complexes sont de taille comparable, et leur plus grand axe est d'environ $12 \mathrm{~km}$.

La caldeira d'Acıgöl a été reconnue pour la première fois par Yildirim et Özgür (1981). Druitt et al. (1995) en ont étudié les produits d'émission et ont proposé une chronologie de son fonctionnement. Le complexe du Göllï Dag n'a pas encore fait l'objet d'étude d'ensemble, et l'identification de sa caldeira dans le présent article est un résultat nouveau important. 
Les recherches réalisées sur les obsidiennes ont permis de dater le fonctionnement post-caldeira des deux complexes (Tableau 1). Les obsidiennes du Göllü Dag datent du Pléistocène moyen alors que celles d'Acıgöl sont datées du Pléistocène supérieur (Bigazzi et al., 1993 et 1997, Chataigner et al., 1998).

Afin de reconstituer le fonctionnement des deux complexes rhyolitiques, nous envisageons successivement trois phases, pré-, syn- et post-caldeira. Chacune est associée à des changements des environnements d'ampleur et de rythme différents, selon le volume des produits émis, leur mode de mise en place et la durée des éruptions.

\section{1 - L'activité pré-caldeira du secteur du Göllï Dag}

Les coupes du complexes d'Acıgöl ne permettent pas d'observer une éventuelle phase pré-caldeira. A l'inverse, on observe dans le complexe du Göllü Dag des horizons téphriques remaniés dans les formations fluviolacustres pré-caldeira. A l'ouest du complexe, on observe, surmontant les ignimbrites néogènes, une série de formations fluvio-lacustres de plus de 20 mètres d'épaisseur (fig. 4A). Elles comprennent des niveaux à galets pouvant atteindre $15 \mathrm{~cm}$ de grand axe, dont le cortège pétrographique est exclusivement composé d'andésites et de basaltes. Ces niveaux dont les structures sédimentaires (lentilles, stratification oblique) et la granulométrie grossière indiquent une forte activité alluviale, témoignent d'une importante phase d'érosion, responsable d'un démantèlement des strato-volcans néogènes. Les coupes présentent une interstratification de ces dépôts avec des faciès lacustres comportant des niveaux de diatomites.

Deux types de téphras remaniés sont présents au sommet de cette formation : il s'agit d'une part de lapilli ponceux porphyriques émoussées et d'autre part de tuffites cendreuses riches en minéraux. Le niveau à ponces associe par ordre d'importance relative, des orthopyroxènes, des amphiboles et des clinopyroxènes (ainsi que de rares minéraux opaques et micas noirs). Les tuffites associent des micas noirs et des minéraux opaques à des clinopyroxènes, des amphiboles et de rares zircons. Dans les deux cas les verres sont rhyolitiques, avec plus de $76 \%$ de $\mathrm{SiO}_{2}$. Cependant la chimie des verres des cendres recouvre le champs des verres du Hasan Dag, alors que celle des verres des ponces est très proche des autres échantillons du Göllü Dag (fig. 5A). Le niveau à ponces témoigne d'une première activité pyroclastique du complexe du Göllü Dag. Les tuffites indiqueraient la contemporanéité entre cette activité et celle du Hasan Dag, déjà suggérée par Pastre et al. (1998).

\section{2 - L'activité syn-caldeira : \\ mise en place des écoulements pyroclastiques \\ Le complexe du Göllï Dag}

Le site 15 (fig. 3 et 4B) permet d'observer la séquence la plus complète des pyroclastites du Göllü Dag. L'unité 2 est une coulée de cendres comportant quelques lapilli ponceux très bien roulés et de très rares lithiques (rhyolites). Sa partie supérieure est enrichie en ponces (unité 3). Le cortège des minéraux lourds a été établi par comptage d'environ 300 grains au microscope polarisant. Il associe des orthopyroxènes, des amphiboles (environ $25 \%$ chacun) des clinopyroxènes $(20 \%)$. Les autres grains sont des minéraux opaques (essentiellement de la magnétite) ainsi que de rares micas noirs et zircons. Cette unité atteint une vingtaine de mètres d'épaisseur au maximum. Elle est absente des coupes les plus occidentales à une douzaine de kilomètres du centre du complexe, mais on peut l'observer dans les coupes les plus orientales (site 17, unité 2).

L'unité 6 est une coulée à cendres et lapilli ponceux dont les ponces à vésicules allongées (faciès pseudofibreux) peuvent atteindre $15 \mathrm{~cm}$ de grand axe. Les lithiques sont constitués d'une rhyolite perlitique à obsidienne, et de rares laves altérées (andésite et basalte). Le cortège des minéraux lourds est constitué par ordre d'importance par les clinopyroxènes et les orthopyroxènes (environ $30 \%$ chacun), puis par des minéraux opaques. Ensuite viennent les amphiboles (environ $10 \%$ ) et des micas noirs. Cette unité peut atteindre une dizaine de mètres d'épaisseur. Elle n'a été observée que dans la partie occidentale du complexe du Göllü Dag.

L'unité 8 est une coulée à cendre et lapilli ponceux dont les ponces, fibreuses à pseudo-fibreuses, atteignent $10 \mathrm{~cm}$ de grand axe. Ce matériel est aphyrique et la séparation densimétrique a livré moins de $0,1 \%$ de minéraux lourds, constitué par quelques micas noirs. Les obsidiennes sont abondantes parmi les lithiques qui comprennent également des blocs de rhyolite et de rares basaltes. Cette formation est présente dans la partie occidentale du complexe où elle atteint une quinzaine de mètres au maximum.

Ces coulées sont interstratifiées avec des déferlantes et des retombées. Ainsi l'unité 4 (log 15 et 17, fig. 4A) est une retombée ponceuse à ponces porphyriques à faciès moussu comportant des quartz, feldspaths et ferromagnésiens (micas noirs et hornblende). Les lithiques sont des lapilli de rhyolite et de lave sombre altérée, ainsi que de rares obsidiennes. En situation proximale (site 18), cette formation atteint $6 \mathrm{~m}$ d'épaisseur. Ces unités pyroclastiques présentent parfois des faciès de remaniement alluviaux (faciès lenticulaires des unités 5 et 7 du $\left.\log \mathrm{n}^{\circ} 15\right)$; cependant la grande homogénéité pétrographique comme l'absence de paléosol indiquent l'absence de répit prolongé dans l'accumulation des pyroclastites syn-caldeira. On observe également une diminution générale des épaisseurs des unités pyroclastiques du centre du complexe vers sa périphérie. En outre, dans la partie occidentale du complexe les unités pyroclastiques fossilisent le réseau hydrographique pré-caldeira, et semblent avoir été canalisées par celui-ci en direction du sud (plaine de Çiftlik).

\section{Le complexe d'Acıgöl}

Les formations syn-caldeira du complexe rhyolitique d'Acıgöl sont particulièrement bien développées à l'est de la caldeira dans le secteur du Kumtepe (fig. 2A). Elles ont été décrites et interprétées par Druitt et al. (1995). Ces auteurs distinguent une série de pyroclastites infé- 

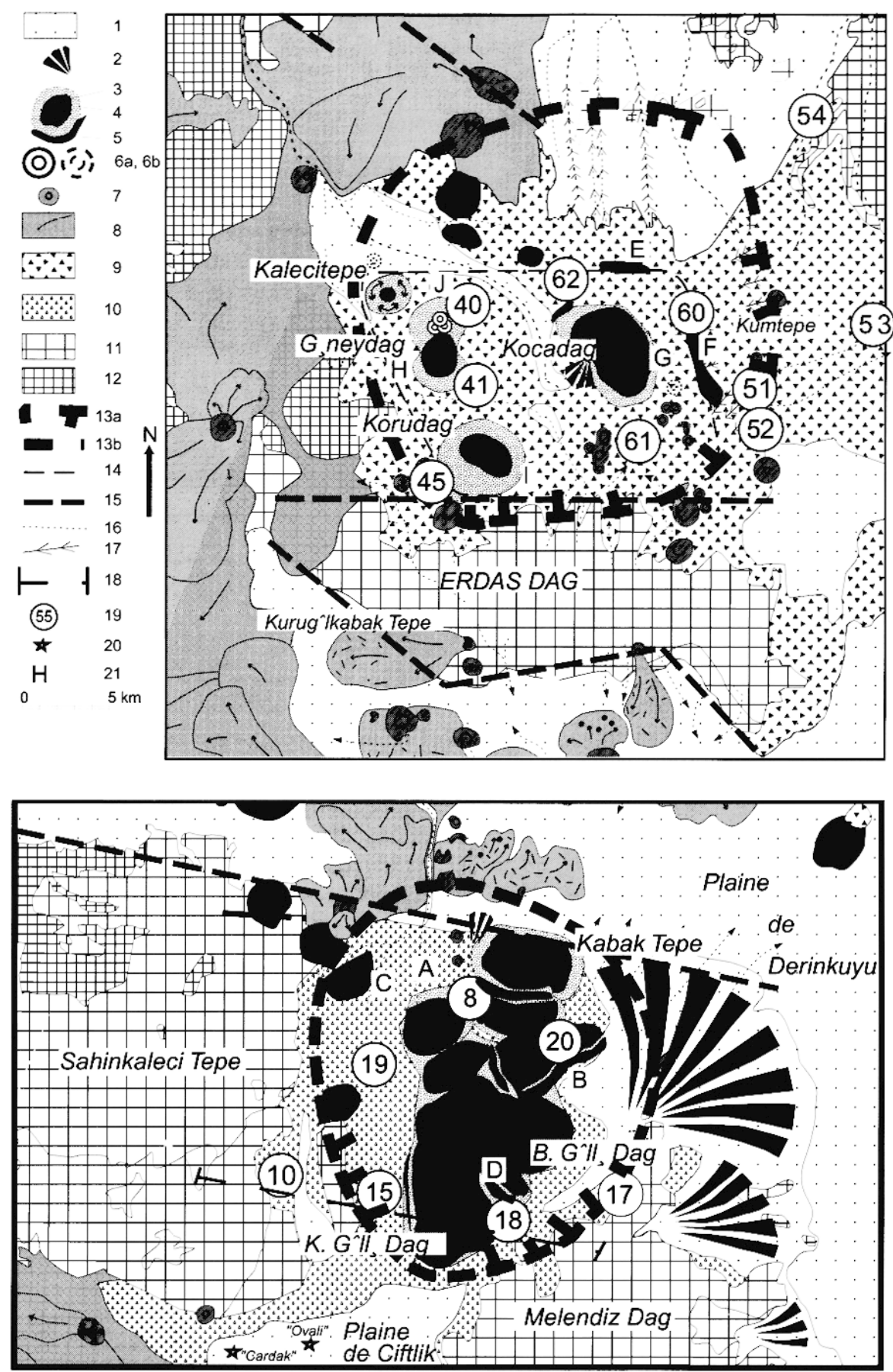

Fig. 2A : Le complexe rhyolitique d'Acıgöl - Acıgöl rhyolitic complex.

Fig. 2A : Le complexe rhyolitique du Göllü Dag - Göllü Dag rhyolitic complex.

1 -alluvions ; 2 -cône alluvial ; 3 -dôme rhyolitique ; 4 - anneau de tuf ; 5 -dyke annulaire d'obsidienne ; 6a - maar ; 6b-maar masqué ; 7 - cône strombolien et cratère ; 8 - coulée basaltique ou andésitique pléistocène, et direction d'écoulement ; 9-pyroclastites syn-caldeira du complexe d'Acıgöl ; 10 - pyroclastites syn-caldeira du complexe du Göllü ; 11 - volcanisme neogène ; 12 - substrat non volcanique ; 13a - limite des caldeiras d'Acıgöl et du Göllü Dag ; 13b - limites masquées ou supposées des caldeiras ; 14 - limite de la caldeira d'Acıgöl d'après Yıldırım and Özgür, 1981 ; 15 - principales failles ;

16 - oued ; 17 -ravins ; 18 - Localisation de la figure $3 ; 19$ - localisation des coupes ; 20 - localisation des carottes " Çardak " et " Ovalı" ; 21 - Localisation des dates TF sur obsidienne (tab. 1).

1 - alluvium ; 2 - alluvial fan ; 3-rhyolitic dome ; 4-tuff ring ; 5-annular obsidian dyke ; $6 a$ - maar ; $6 b$ - hidden maar ; 7 stombolian cone and crater ; 8-Pleistocene basaltic or andesitic lava, and flow direction; 9 - Acıgöl complex : syn-caldera pyroclastites ; 10 - Göllü Dag complex : syn-caldera pyroclastites ; 11-Neogene volcanism; 12 - non-volcanic substratum ; $13 a$ - limits of Actgöl and Göllü Dag calderas ; 13b-hidden limits of calderas ; 14 - Actgöl caldera limit according to Yildirım and Özgür, 1981; 15-Main fault ; 16-wadi ; 17-gully;18-Location of cross section, fig. $3 ; 19-$ Location of sections ; 20 - Location of " Çardak" and "Ovall" cores - 21 - Location of fission track dates on obsidian (tab. 1). 


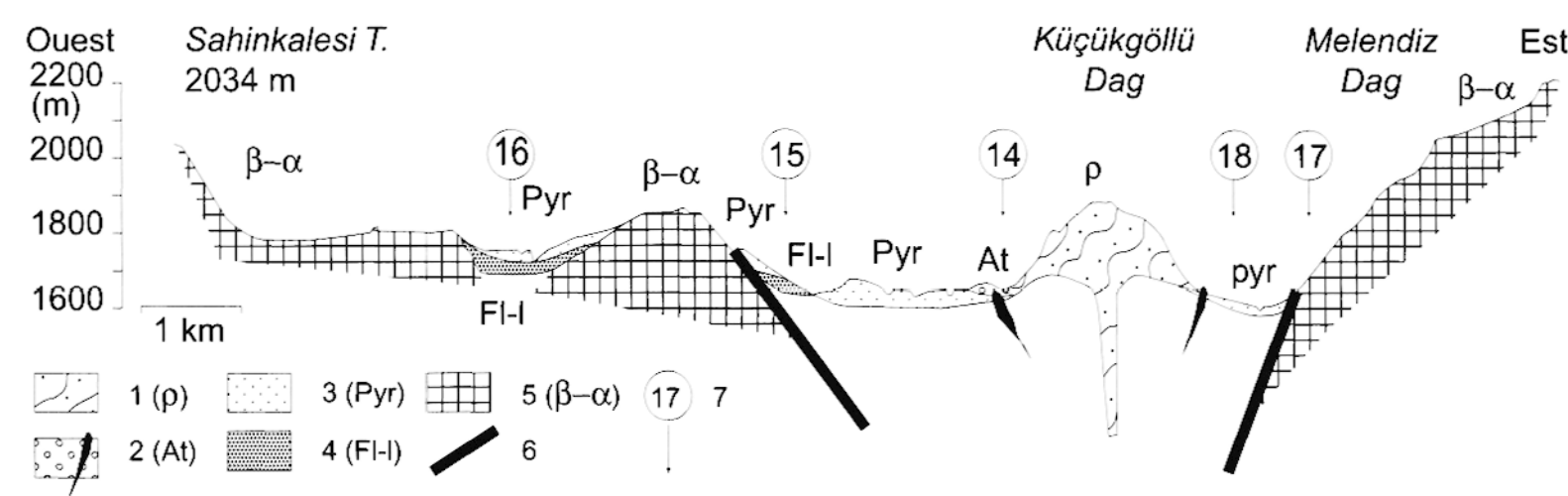

Fig. 3 : Coupe à travers le complexe du Göllü Dag.

$1(\rho)$ - Dôme rhyolitique ; 2 (At) - Anneau de tuf et dyke annulaire d'obsidienne ; 3 (Pyr) - Pyroclastites syn-caldeira ; 4 (Fl-l)

- Formations fluvio-lacustres ; $5(\beta-\alpha)$ - Volcanisme anté-quaternarie ; 6 - Failles ; 7 - Localisation des logs de la figure $4 \mathrm{~A}$.

Fig. 3 : Cross section of the Göllï Dag complex.

$I(\rho)$ - Rhyolitic dome ; $2(A T)$ - Tuf ring and annular obsidian dyke ; 3 (Pyr)-Syn-caldeira pyroclastites ; 4 (Fl-l) - Fluviolacustrine formation ; $5(\beta-\alpha)$-Pre-quaternary volcanism ; 6 -Fault ; 7 -Location of logs, fig. $4 A$.

rieures comportant les principales coulées, et une série supérieure comprenant essentiellement des retombées pliniennes et des déferlantes.

Les nouvelles coupes observées à proximité et sur le Kumtepe ne permettent pas de distinguer cette succession : les pyroclastites attribuées à la série supérieure précèdent celles attribuées à la série inférieure. Les pyroclastites contemporaines de la formation de la caldeira procèdent d'une succession très rapide, comme l'indique l'absence de formation alluviale, colluviale ou de paléosol interstratifié. Au site $n^{\circ} 51$ (fig. 4B), les formations syn-caldeira atteignent une trentaine de mètre d'épaisseur. L'activité pyroclastique débute par la mise en place d'une retombée plinienne à ponces aphyriques à faciès fibreux tubulaire (unite 1). Parmi les lithiques, on note l'absence d'obsidienne, qui est présente dans les unités supérieures. Cette retombée est recouverte par une épaisse série de coulées à cendres et lapilli ponceux (unités 2 et 6) dans laquelle viennent s'intercaler des déferlantes (unité 3), une retombée cendreuse (unité 4) et un lag breccia (unité 5). Après une nouvelle série de déferlantes (unité 8), le régime plinien domine de nouveau (unités 9 à 13). Au site 53 , l'unité 1 fossilise une paléovallée à l'intérieur de laquelle les écoulements pyroclastiques postérieurs viennent s'emboîter, expliquant que ces écoulements peuvent, localement, se situer en position altitudinale inférieure. En effet, les trois coulées pyroclastiques de l'unité 2 ont été canalisées en direction du nord-nord-est, par un réseau hydrographique préfigurant le système de la Kızılırmak. Elles prennent de l'ampleur en direction de Nevsehir où elles atteignent 20 mètres d'épaisseur (site $n^{\circ} 54$ ).

Les nouvelles analyses effectuées sur l'ensemble des échantillons des unités 1 à $21(\log 51)$ confirment l'homogénéité géochimique de l'ensemble des pyroclastites syn-caldeira, avec une teneur en $\mathrm{SiO}_{2}$ comprise entre 71,7 et $72,7 \%$. En revanche, l'activité post-caldeira, qui apparait sous forme d'une retombée à lapilli de ponce d'environ $70 \mathrm{~cm}$ d'épaisseur (unité $11, \log 52$ ) se distingue nettement des unités syn-caldeira (fig. 5A).

\section{Extension et volumes des pyroclastites}

Les coulées pyroclastiques du Göllü Dag, comme celles d'Acıgöl témoignent d'éruptions majeures. L'ensemble des pyroclastites du complexe d'Acıgöl recouvre une surface de près de $1000 \mathrm{~km}^{2}$ et représente un volume de $28,2 \mathrm{~km}^{3}$ (Druitt et al., 1995).

Les pyroclastites du complexe du Göllü Dag recouvrent actuellement une superficie beaucoup plus modeste, d'environ $150 \mathrm{~km}^{2}$ (tableau 2). Cependant, deux indices témoignent d'une extension originelle plus grandes de ces pyroclastites qui participent au comblement des plaines de Derinkuyu et de Çiftlik. D'une part, des ponces sont remaniées dans les cônes alluviaux bordant le Melendiz $\mathrm{Da}_{-}$, au sud de la plaine de Çiftlik. Il $\mathrm{s}$ 'agit de ponces roulées de 1 à $2 \mathrm{~cm}$ de diamètre maximum, renfermant quelques quartz et feldspaths ainsi que de rares ferromagnésiens, associés à quelques grains d'obsidiennes $(<5 \%)$. Cette formation alluviale indique que les pyroclastites du Göllü Da_recouvraient initialement une grande partie du Melendiz Dag. De plus, des horizon téphriques ont été retrouvés dans deux carottes prélevées au nord de la plaine de Çiftlik (fig. 2B) à 765 et $865 \mathrm{~cm}$ de profondeur (carotte "Çar") et à $705 \mathrm{~cm}$ (carotte "Ovali)". Ces horizons apparaissent au sein d'une sédimentation non continue, de type fluviolacustre faisant alterner des niveaux grossiers (alluvions) et des niveaux marneux ou de diatomites. Les horizons téphriques comprennent des ponces de la taille des sables moyens, associées à des grains de quartz et à des biotites. Les analyses géochimiques (fig. 5A) montrent une très grande proximité de composition entre ces échantillons, et ceux issus de l'activité syn-caldeira du Göllü Dag.

En englobant le complexe du Göllü Dag, la partie nord du Melendiz Dag, la moitié orientale du gahinkalesi Tepe ainsi que la plaine de Çiftlik et une partie de celle de Derinkuyu, l'extension originelle des produits syncaldériques peut être estimée à plus de $720 \mathrm{~km}^{2}$. En admettant une épaisseur moyenne de $30 \mathrm{~m}$, le volume correspondant est de $21,7 \mathrm{~km}^{3}$ (tableau 2). 


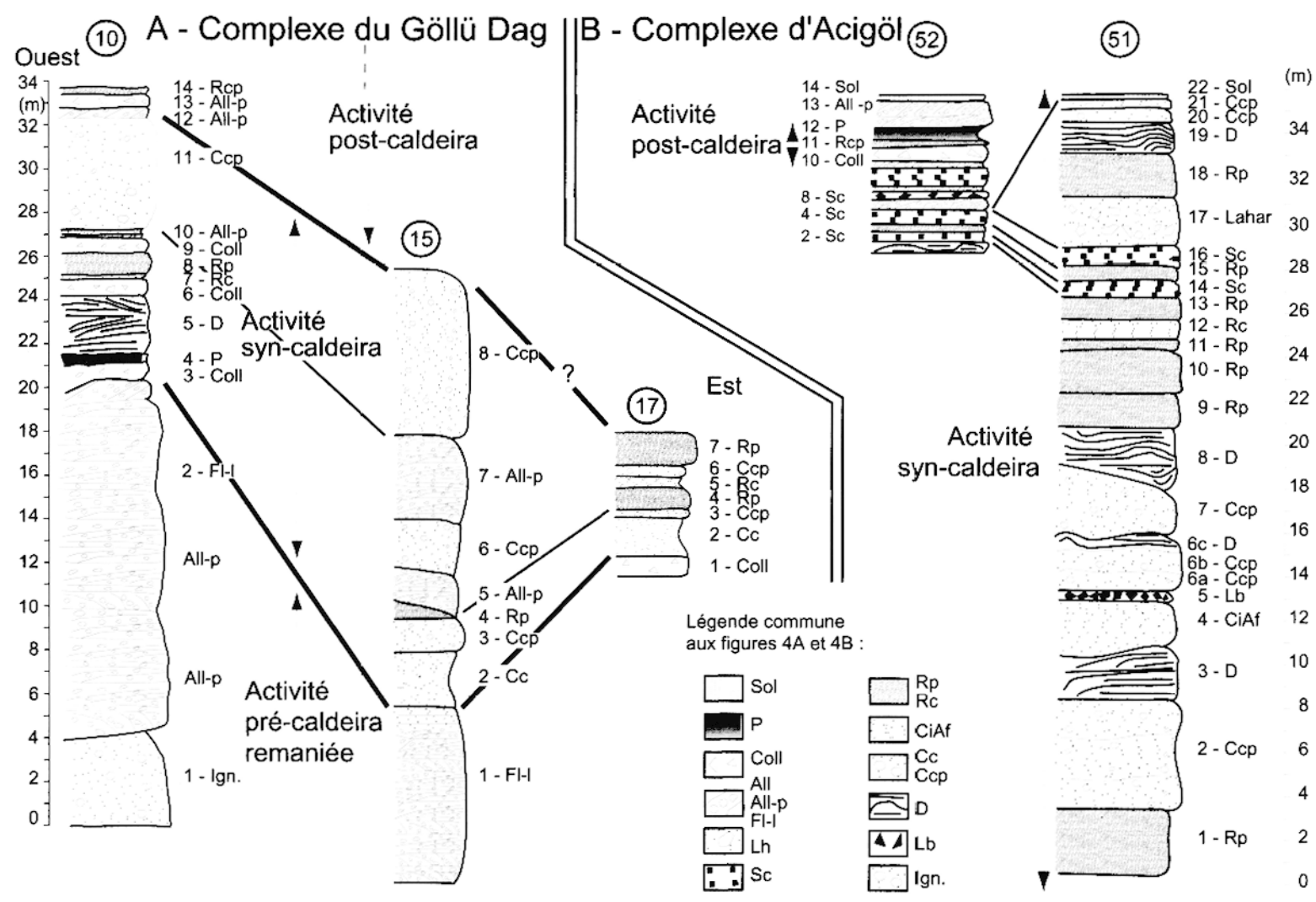

Fig. 4A : Formations pyroclastiques associées à la mise en place du complexe rhyolitique du Göllü Dag. Fig. 4 A: Pyroclastites from Göllü Dag rhyolitic complex.

Figure 4B : Kumtepe : formations orientales du complexe d'Acigöl.

Fig. $4 B$ : Kumtepe : eastern formations of Acıgöl complex.

Figures 4A et 4B : S - sol ; P - paléosol ; Coll - colluvions ; All - alluvions ; All-p - alluvions ponceuses ; Fl-l - formations fluviolacustres ; $\mathrm{Lh}$ - lahar ; $\mathrm{Sc}$-scories basaltiques ; $\mathrm{Rp}$ - retombée ponceuse ; Rc-retombée cendreuse ; Ciaf - retombée co-ignimbritique ; $\mathrm{Cc}$ - coulée cendreuse ; $\mathrm{Ccp}$-coulée cendro-ponceuse ; D - déferlantes ; Lb - brèche ; Ign - ignimbrite néogène.

Figures $4 A$ and $2 B: S$-soil ; $P$-paleosol ; Coll-colluvium ; All-alluvium ; All-p-pumice-rich alluvium ; Fl-l-fluvio-lacustrine formations; $L h$-lahar ; $S c$ - basaltic scoriae ; $R p$ - pumice fall ; Rc-ash fall ; Ciaf-co-ignimbritic ash fall; Cc-ash flow ; $C c p$ - ash and pumice flow ; D-surge; Lb-lag breccia; Ign-Neogene ignimbrites.

\section{3 - L'activité syn-caldeira : \\ l'effondrement des systèmes caldeiriques}

\section{La caldeira du Göllii Dag}

Les coupes occidentales du complexe du Göllü Dag permettent d'observer l'effondrement du centre du complexe rhyolitique corrélatif à la mise en place des pyroclastites. La coupe du site 15 montre le sommet de la sédimentation fluvio-lacustre et le contact entre des tuffites et des alluvions à galets d'andésite et de basalte. L'inclinaison vers l'est de cette formation (environ $33^{\circ}$ ), témoigne de l'effondrement du centre de la structure. Le pendage des formations sus-jacentes présente une direction identique, mais une inclinaison moindre $\left(15^{\circ}\right.$ environ pour le contact entre les unités 7 et 8 ) qui indique un ralentissement de la subsidence. Cette observation, comme la présence d'unités pyroclastiques faiblement remaniées interstratifiées entre les deux coulées pyroclastiques principales, suggère un fonctionnement polyphasé de la caldeira. Ces coupes soulignent la limite occidentale de la caldeira du Göllü Dag, au contact du Sahinkalesi Tepe. De même la limite entre cette caldeira et le massif du Melendiz Dag correspond à un escarpement bien visible.

Au contraire, les limites nord-est et sud n'apparaissent pas dans la topographie. Au nord-est, se situe la plaine de Derinkuyu, alors qu'au sud, se trouve celle de Çiftlik. Il s'agit de deux graben (Dhont et al., 1998), pressentis comme d'anciennes caldeiras à l'origine des grandes ignimbrites néogènes (Le Pennec et al. 1994). Ainsi définie, la caldeira du Göllü Dag a une superficie d'environ $113 \mathrm{~km}^{2}$, et un volume de $33,9 \mathrm{~km}^{3}$, pour un effondrement estimé à $300 \mathrm{~m}$.

\section{Délimitation de la caldeira d'Acıgöl}

Yildirim \& Özgur (1981) ont, les premiers, reconnu et cartographié la caldeira d'Acıgöl. Cependant la limite proposée par ces auteurs doit être révisée à la lumière de nos observations. Ce qu'ils définissent comme le "mur" oriental de la caldeira constitué d'obsidienne dont les plans de fluidité sont sub-verticaux correspond en fait à un dyke annulaire relié à l'activité du Kocadag. Ceci explique que ces affleurements n'apparaissent qu'à proximité de ce dôme. La nouvelle limite que nous indi- 
quons sur la figure 2A s'appuie au sud sur l'Erdas dag, et à l'est sur la limite du Kum Tepe où elle définit un talus rectiligne de plus de 50 mètres de hauteur. Au nord et à l'ouest les limites correspondent aux affleurements d'ignimbrites néogènes et englobent l'ensemble des dômes affleurant dans la caldeira. Au nord-ouest, le réseau hydrographique est en partie responsable du démantèlement de cette limite. Ainsi définie, la caldeira a une superficie d'environ $150 \mathrm{~km}^{2}$. Pour une profondeur de $200 \mathrm{~m}$, l'effondrement représente un volume de $30 \mathrm{~km}^{3}$.

\section{4 - L'activité post-caldeira : \\ l'extrusion des dômes rhyolitiques}

Ces extrusions sont mises en places selon un modèle original dans lequel il est possible de repérer quatre phases principales assujetties à des variantes. Dans un premier temps, une activité phréato-magmatique est responsable du fonctionnement de maars et de la mise en place d'anneaux de projections pyroclastiques (Kaleci Tepe au nord-ouest de la caldeira d'Acıgöl, ou Kabak Tepe, au nord de la caldeira du Göllü Dag, site $n^{\circ} 8$ ). Cette première activité est parfois de type phréato-plinienne (site ${ }^{\circ} 41$, Güneydag, dans la caldeira d'Acıgöl). Ensuite, des coulées à cendres et blocs indiquent une évolution vers un régime de type péléen (Kabak Tepe, site ${ }^{\circ} 8$ ), et l'écroulement d'un premier dôme. On observe parallèlement la mise en place de dykes annulaires de perlite et d'obsidienne à la périphérie des anneaux de tuf (à l'est du Kocadag, site $n^{\circ} 60$ ou à Kaletepe au site $n^{\circ} 10$, par exemple). Ces dykes montrent des structures de fluidité sub-verticales, présentant généralement une transition entre la partie centrale du dyke constituée d'une obsidienne très vitreuse (qui constitue la matière première la plus adaptée à la taille d'outils préhistoriques) et la partie périphérique de plus en plus perlitique vers les bords (par exemple sur le flanc nord du Büyük Göllü Dag, ou à l'est du Kocadag). Ces dykes annulaires ont fourni les échantillons datés par trace de fission et $\mathrm{Ar} / \mathrm{Ar}$ (tableau 1, Bigazzi et al., 1993 et 1997, Chataigner et al. 1998). Puis le dôme terminal lui-même est extrudé à l'intérieur de l'ensemble précédent.

Les dykes annulaires d'obsidienne correspondent probablement à des injections concentriques bordant les structures de maar. Il sont généralement incomplet et ne sont pas visibles autour de tous les dômes. Ainsi, en ce qui concerne la caldeira d'Acıgöl, le Korudag, le Güneydag, et le Kaleci Tepe qui sont les édifices les plus jeunes (les deux premiers ont été datés de $20 \mathrm{ka}$ par Bigazzi et al., 1993) et les plus petits, ne présentent pas de dyke annulaire d'obsidienne visible. Au contraire, le dôme du Kocadag (environ 180 ka, tabl. 1) présente un dyke annulaire visible à l'est et au nord de l'édifice (sites 60 et 61 ). Les premières observations indiquent donc que les dykes annulaires d'obsidienne sont préférentiellement localisés autour des édifices les plus grands et les plus anciens.

Ce modèle est récurrent, bien que chacune des quatre phases décrites ne soient pas observables pour chaque dôme. Ainsi, on compte 9 dômes à l'intérieur du complexe du Göllü Dag, et 5 dans celui d'Acıgöl. Les dômes
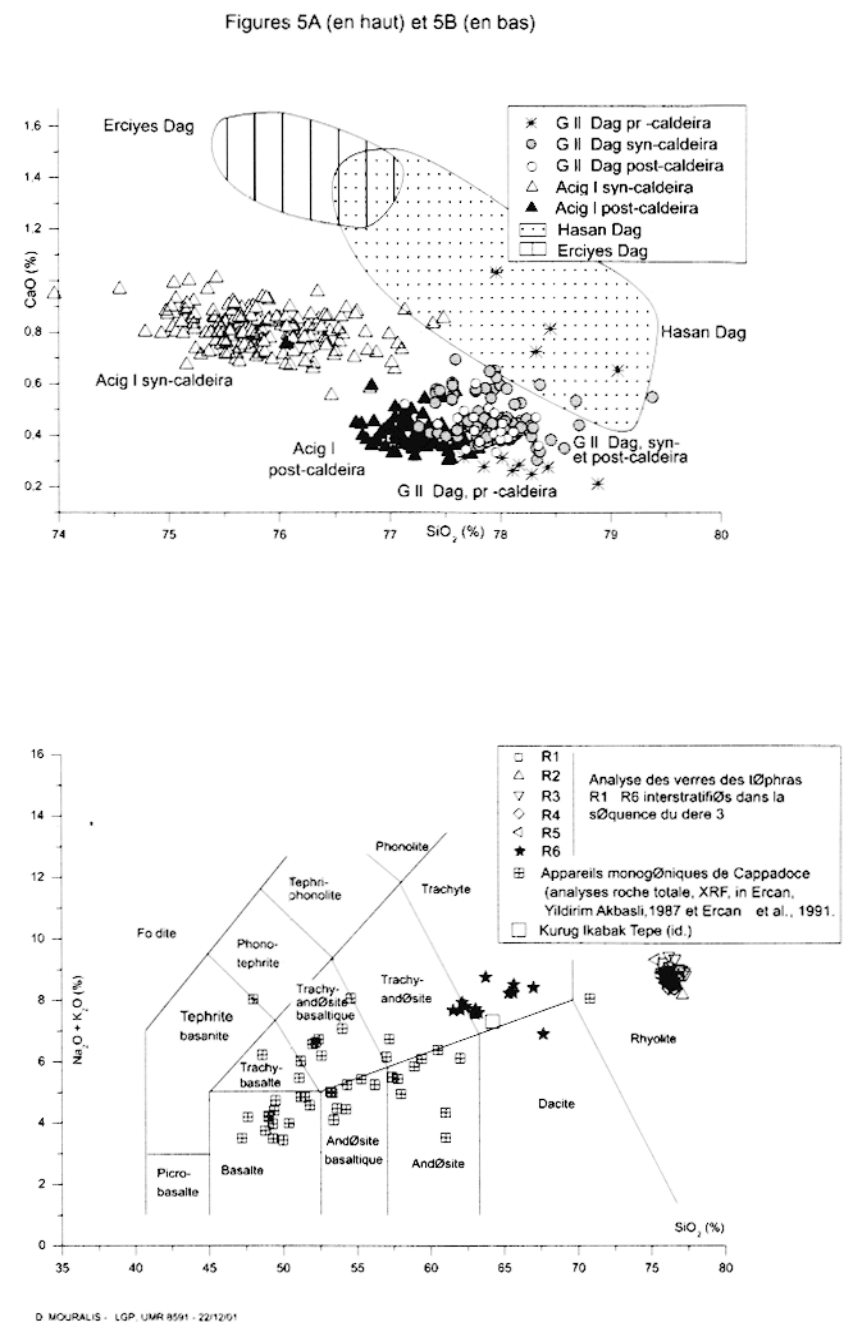

Fig. 5A : Géochimie des verres de quelques téphras des complexes du Göllü Dag et d'Acıgöl et comparaison avec des produits du Hasan Dag et de l'Erciyes Dag.

Fig. 5A: Glass geo-

chemistry of some tephras from Göllü Dag and Acıgöl complexes and comparison with volcanic products from Hasan Dag and Erciyes Dag.

Figure 5B : Géochimie des verres des téphras interstratifiés dans la séquence du " Dere 3 " de Kaletepe (site ${ }^{\circ}{ }^{20}$ ) comparée à celles de quelques appareils monogéniques proches (diagramme TAS, d'après Le Bas et al., 1984).

Fig. 5B : Glass geochemistry of tephras in the "Dere 3 " section, from Kaletepe (section $n^{\circ} 20$ ) and comparison with some close monogenic volcanoes (TAS diagram, from Le Bas et al., 1984).

Figure 5A \& B, conditions d'analyse. Opérateur : D. Mouralis - Microsonde Cameca SX 100 - Laboratoire " Magmas et

Volcans ", Clermont-Ferrand - Faisceau défocalisé à $10 \mu \mathrm{m}$; tension $15 \mathrm{kv}$; intensité $6 \mathrm{nA}$; temps de comptage $10 \mathrm{~s}-$ Valeurs normalisées à $100 \%$.

Figure $5 A \& B$, analyze conditions. Operator : D. Mouralis Microprobe Cameca SX 100 - "Magmas et Volcans " laboratory, Clermont-Ferrand - Beam defocalised to $10 \mu \mathrm{m}$; tension $15 \mathrm{kv}$; intensity $6 \mathrm{nA}$; count time $10 \mathrm{~s}-100 \%$ normalized results. 


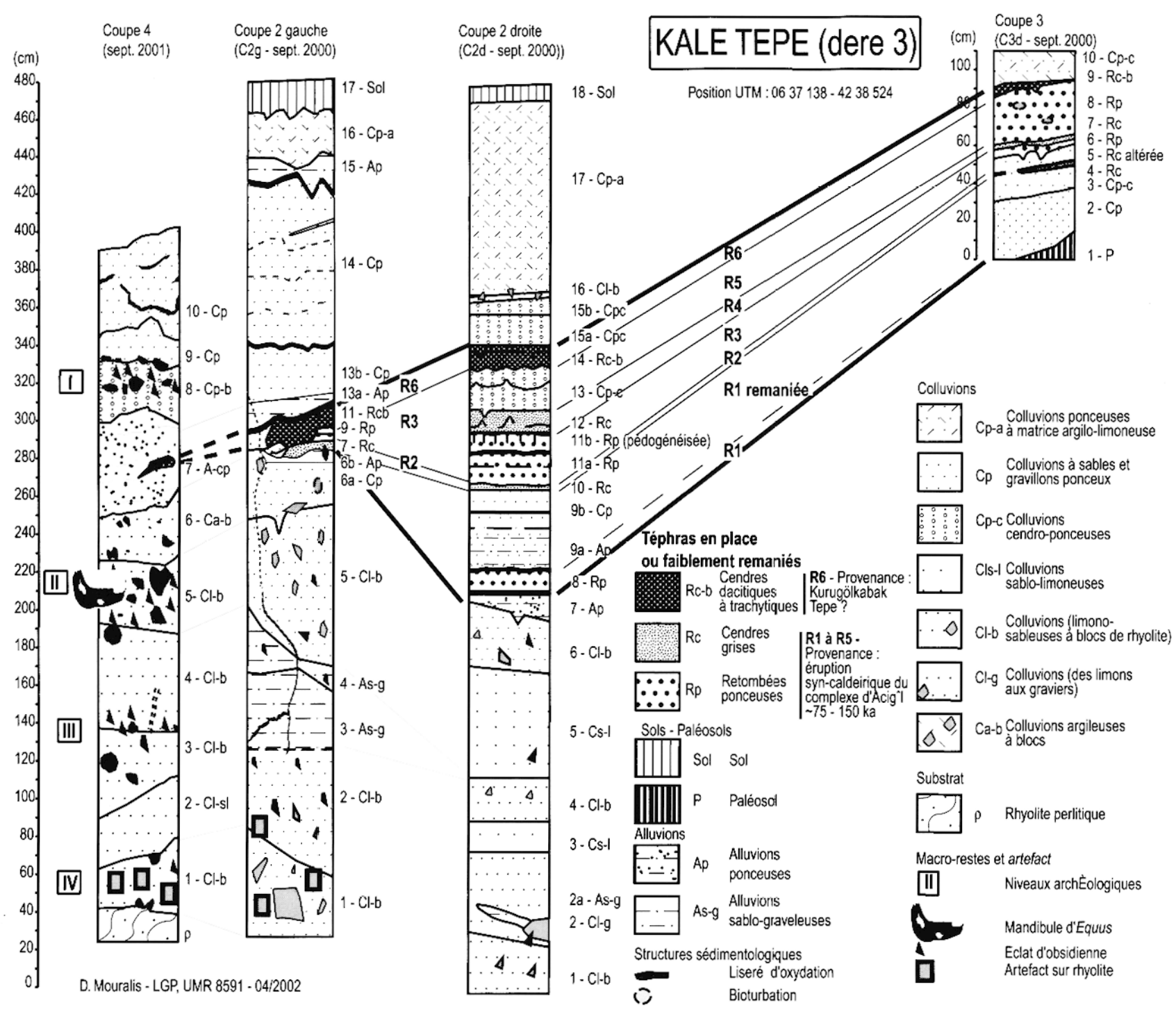

Fig. 6 : Téphras interstratifiés dans la coupe du " Dere 3 " (site n²0).

Fig. 6 : Kale Tepe, complexe du Göllü Dag. - Tephra in "Dere 3" section ( $\left.n^{\circ} 20\right)$-Kale Tepe, Göllü Dag complex.

et pyroclastites post-caldeira du complexe d'Acıgöl représentent un volume total de $2,3 \mathrm{~km} 3$, alors que ceux du complexe du Göllü Dag atteignent $15 \mathrm{~km} 3$ (volumes calculés grâce à un modèle numérique de terrain réalisé à partir des cartes topographiques au $1: 25000$ ).

\section{5 - L'activité post-caldeira : interstratification de téphras et de matériel archéologique}

Les phases post-caldeira correspondent à une activité volcanique plus étalée dans le temps que celle des phases syn-caldeira, mais beaucoup moins importante en ce qui concerne les volumes de pyroclastites émis et les changements environnementaux induits. Ces conditions ont permis l'interstratification des pyroclastites avec des sédiments alluviaux et colluviaux, ainsi que le développernent de palésols.

La coupe du Dere 3 de Kaletepe ( ${ }^{\circ} 20$, fig. 6) montre ainsi l'interstratification de six niveaux archéologiques (Slimak et Roche, 2001), de six téphras et de différents niveaux colluviaux et alluviaux. Parmi les quatre niveaux archéologiques dont la fouille a débuté en septembre 2001, le plus ancien (niveau IV) s'individualise des trois suivants par la matière première utilisée, composée quasi-exclusivement de rhyolite. La présence de quelques pièces pouvant se rapprocher d'un débitage Levallois permet de le rapporter au Paléolithique moyen ancien. Les niveaux supérieurs (III à I), moustériens, incorporent essentiellement des artefacts sur obsidienne. Le niveau II comprend des éléments caractéristiques d'un débitage Levallois, à tendance parfois laminaire. Ce niveau a également livré une mandibule d'Equus bien conservée. Le niveau supérieur (I) présente lui aussi des pièces s'inscrivant dans une chaîne opératoire Levallois.

Six téphras sont présents entre les niveaux I et II. Le téphra supérieur (R6) est de couleur sombre, cendreux comprenant des grains scoriacés et présente une composition dacitique à trachytique $(\mathrm{SiO} 2=63,9 \%$ et 


\begin{tabular}{|l|l|l|l|l|}
\cline { 2 - 5 } \multicolumn{1}{c|}{} & \multicolumn{2}{c|}{ Göllü Dag } & \multicolumn{2}{c|}{ Acıöl } \\
\cline { 2 - 5 } \multicolumn{1}{c|}{} & \multicolumn{1}{c|}{$\begin{array}{c}\text { Superficie } \\
\left(\mathrm{km}^{2}\right)\end{array}$} & $\begin{array}{c}\text { Volume } \\
\left(\mathrm{km}^{3}\right)\end{array}$ & $\begin{array}{c}\text { Superficie } \\
\left(\mathrm{km}^{2}\right)\end{array}$ & $\begin{array}{c}\text { Volume } \\
\left(\mathrm{km}^{3}\right)\end{array}$ \\
\hline Caldeira & 113 & 33,9 & 150 & 30 \\
\hline Pyroclastites syn-caldeira & $\begin{array}{l}\left(150^{(*)}\right) \\
720\end{array}$ & 21,7 & $1000^{(* *)}$ & $28,2^{(* *)}$ \\
\hline $\begin{array}{l}\text { dômes et pyroclastites } \\
\text { post-caldeira }\end{array}$ & 51,9 & 12,5 & 21,2 & 2,3 \\
\hline
\end{tabular}

" extension actuelle des pyroclastites, et extension originelle estimée.

${ }^{\prime \cdots}$ sources : Druitt et al., 1993. Les autres résultats proviennent de la présente étude.

Tab. 2 : Superficies et volumes des caldeiras et des pyroclastites associées

Tab. 2 : Area and volume of caldeiras and associated pyroclastites.

$\mathrm{Na} 2 \mathrm{O}+\mathrm{K} 2 \mathrm{O}=7,9 \%$, fig. 5B). La comparaison avec les données géochimiques publiées concernant la plupart des appareils monogéniques de la région (Ercan, Yildirim et Akbasl, 1987, et Ercan et al., 1991) permet d'attribuer ce téphra au Kurugölkabak Tepe, cône strombolien situé à environ $15 \mathrm{~km}$ au nord du Göllü Dag (fig. 2A). Les cinq téphras inférieurs sont ponceux (R1, R3, R4 et R5) ou cendreux (R2) et ont une composition rhyolitique ( $\mathrm{SiO} 2$ compris entre 75,8 à 76,5 et $\mathrm{Na} 2 \mathrm{O}+\mathrm{K} 2 \mathrm{O}$ entre 8,6 et $8,9 \%$, fig. 5B).

Afin de localiser l'origine des téphras, nous avons comparé les résultats des analyses chimiques, avec notre base de données. Celle-ci comporte les analyses des majeurs d'une centaine d'échantillons que nous avons prélevés en situation proximale et provenant d'édifices volcaniques actifs au cours du Pléistocène moyen et supérieur (Hasan Dag, Erciyes Dag, Acıgöl et Göllü Dag). Cette comparaison montre la grande proximité entre les téphras du Dere 3 et ceux provenant de l'activité syn-caldeira d'Acigöl (fig. 5A).

L'analyse des téphras interstratifiés entre les niveaux archéologiques I et II permet ainsi de disposer de jalons chronologiques. Les cinq téphras rhyolitiques se rapportant à l'activité syn-caldeira d'Acıgöl ont un âge postérieur à $180 \mathrm{ka}$ (âge des obsidiennes qu'elles remanient) et antérieur à $75 \mathrm{ka}$ (âge des obsidiennes qui les fossilisent) (Bigazzi et al., 1993, Druitt et al., 1995). Cette fourchettc est tout à fait cohérente avec les caractéristiques technologiques des artefacts des niveaux archéologiques I et II qui encadrent ces téphras.

\section{DISCUSSION ET CONCLUSIONS}

Si les limites des deux caldeiras n'apparaissent pas partout dans la topographie, différents arguments permettent d'étayer leur existence. Tout d'abord, morphologiquement, des escarpements forment une dépression semi-circulaire au sud-est du complexe d'Acıgöl. Dans le complexe du Göllü Dag, les limites entre ce massif et ceux du Melendiz. Dag et du Sahinkalesi Tepe sont nettement visibles. En outre, le basculement des alluvions anté-caldériques du complexe du Göllï Dag témoigne de l'effondrement de la partie centrale de la structure. Dans les deux cas, cet effondrement est également indiqué par la répartition des affleurements des roches pré-quaternaires. Le substrat anté-volcanique et les laves néogènes entourent les complexes, mais sont absents des parties centrales. En outre, les grandes nappes pyroclastiques associées à ces structures se sont écoulées de manières divergentes par rapport au centre des complexes.

De plus, dans le cas du complexe d'Acıgöl, le volume effondré de la caldeira que nous avons définie (30 $\left.\mathrm{km}^{3}\right)$ correspond au volume de pyroclastites $\left(28,2 \mathrm{~km}^{3}\right)$ calculé par Druitt et al. (1995). En revanche, le volume de pyroclastites du Göllü Dag $\left(22 \mathrm{~km}^{3}\right)$ est bien inférieur au volume de la caldeira $\left(34 \mathrm{~km}^{3}\right)$. Cette différence peut s'expliquer par l'érosion des formations syn-caldériques et leur piégeage dans les plaines de Derinkuyu et de Çiftlik. Il est également intéressant de noter que, dans les deux complexes, les volumes totaux des produits syn- et post-caldérique sont très proches des volumes effondrés (tableau 2).

L'étude des deux complexes du Göllü Dag et d'Acıgöl permet de préciser l'ampleur des changements environnementaux liés au volcanisme explosif rhyolitique quaternaire d'Anatolie centrale. Il est possible de distinguer trois phases dont les conséquences varient en fonction des volumes de pyroclastites émis et de la rapidité des processus. La phase pré-caldeira du Göllü Dag n'a laissé que des traces discrètes sous forme d'horizons téphriques remaniés dans la série fluvio-lacustre. Au contraire, les éruptions syn-caldeira et les effondrements caldeiriques consécutifs ont entraîné la disparition des structures volcaniques néogènes pré-existantes. La mise en place des pyroclastites dont le volume a été estimé de 20 à $30 \mathrm{~km}^{3}$ respectivement pour les complexes du Göllü Dag et d'Acıgöl, a été particulièrement rapide comme l'atteste l'absence de formation alluviale et colluviale, ou de paléosol interstratifié. Enfin, les phases post-caldeira ont entraîné des changements de moindre ampleur marqués par l'extrusion de dômes rhyolitiques et la mise en place d'intrusions d'obsidienne utilisées par les populations paléolithiques puis néolithiques. Les volumes impliqués sont moins importants : $12,5 \mathrm{~km} 3$ dans le complexe du Göllü Dag et seulement $2,3 \mathrm{~km} 3$ dans celui d'Acıgöl. Les âges obtenus sur les obsidiennes (Bigazzi et al., 1993 et 1997, Chataigner et al., 
1998) permettent d'estimer la durée de cette troisième phase : environ $500 \mathrm{ka}$ pour la phase post-caldeira du Göllü Dag, et près de 200 ka pour celle d'Acıgöl. En outre, les nombreuses coupes montrant une interstratification entre les pyroclastites et les formations alluviales et colluviales attestent d'une évolution beaucoup moins rapide que dans la phase précédente.

\section{REMERCIEMENTS}

D. Mouralis bénéficie d'une allocation de recherche MENRT. Cette étude a reçu le soutien financier du Laboratoire de Géographie Physique de Meudon (CNRS, UMR 8591), et du MTA qui s'est également chargé d'une grande partie de la logistique des missions en Turquie. Cette recherche s'inscrit dans le cadre du programme de coopération bilatérale CNRS-TÜBITAK $n^{\circ} 9487$ "Volcanisme et paléogéographie au Pléistocène supérieur en Cappadoce, Turquie ". Elle a bénéficié également de l'aide du programme ECLIPSE du CNRS "Césame" (coord. M. Patterne). Nous remercions A. Gourgaud qui nous a ouvert les portes du service microsonde du laboratoire "Magmas et Volcans" de Clermont-Ferrand. Nous sommes redevables du soutien de la Mission française du Ministère des Affaires Etrangères de Kaletepe et tout particulièrement de Nur Balkan Atli et de Didier Binder. Le auteurs remercient J.M. Cantagrel et E. Juvigné pour leur relecture attentive et leurs remarques constructives. Nous remercions chaleureusement Turgut Yılmaz, Metin et Ugras et toute l'équipe de Jemas Ltd. à Ankara, et Lütfi Dokuzoglu en Cappadoce, pour leur aide et leur soutien permanent.

\section{BIBLIOGRAPHIE}

ATABEY E., (dir.), 1989, Geologic map of the Aksaray il 18 quadrangle, MTA, Carte géologique $1: 100000^{\circ}+$ notice $12 \mathrm{p}$.

AYDAR E., GOURGAUD A, 1998 - The geology of Mount Hasan stratovolcano, Central Anatolia, Turkey. Journal of Volcanology and Geothermal Research, 85, 129-152.

BIGAZZI G., YEGINGIL Z., ERCAN T., ODDONE M., ÖZDOGAN M., 1993 - Fission track dating obsidians in Central and Northern Anatolia. Bulletin of Volcanology, 55, 588-595.

BIGAZZI G., YEGINGIL Z., BOZTUG D., ERCAN T., NORELLI P.., ÖZDOGAN M., 1997 - Studi di provenienza della ossidiana con il metodo delle tracce di fissione : nuovi dati sulle potenziali fonti anatoliche. IV Giormata "Le Scienze della Terra e l'Archeometria ", Naples, 20-21 fevrier 1997, Abstract, p. 33.

CAUVIN M.-C., GOURGAUD A., GRATUZE B., ARNAUD N., POUPEAU G., POIDEVIN J.-L. CHATAIGNER C., (dir.), 1998 - L'obsidienne au Proche et Moyen Orient. Du volcan à l'outil. BAR International Series 738 - Maison de l'Orient Méditerranéen, $388 \mathrm{p}$.

\section{CHATAIGNER C., POIDEVIN J.-L. ARNAUD N. O., 1998}

- Turkish occurrences of obsidian and use by prehistoric peoples in the Near East 14000 to 6000 BP. Journal of Volcanology and Geothermal Research, 85, 517-537.

DHONT D., CHOROWICZ J., YÜRÜR T., FROGIER J.I. KÖSE O., GÜNDO_DU N., 1998 - Emplacement of volcanic vents and geodynamics of Central Anatolia, Türkey. Journal of Volcanology and Geothermal Research, 85, 33-54.

DRUITT T. H., BRENCHLEY P. J, GÖGTEN Y. E., FRANCAVIGLIA V., 1995 - Late Quatemary hyolitic enuption from the Acıgöl Complex, central Turkey. Journal of Geological Society, 152, 655-667.

ERCAN T, AKBASLI A.,YILDIRIM T, FISEKCYI A., SELVI Y, ÖLMEZ M., CAN B., 1991 - Acıgöl (Nevsehir) yöresendeki senozoyik yaslı volkanik kayaçların petrolojisi. Maden Tetkik ve Arama Dergisi, 113, 31-44.
ERCAN T., YILDIRIM T., AKBASLI A., 1987 - Gelveri (Nigde) - Kızılcın (Nevsehir) arasındaki volkanizmanın özllikleri. Jeomorfoloji Dergisi, 15, 27-36.

KELLER J., 1974 - Quaternary maar volcanism near Karapınar in Central Anatolia. Bulletin Volcanologique, 38, 378-396.

LE BAS M. J., LE MAITRE R. W., STRECKEISEN A., ZANATTIN B., 1986 - A chemical classification of volcanic rocks based on the total alcali silica diagram. Journal of Petrology, 27, 745-750.

LE PENNEC J.-L., BOURDIER J.-L., FROGER J. L., TEMEL A., CAMUS G., GOURGAUD A., 1994 - Neogene ignimbrites of the Nevsehir Plateau (Central Turkey) : Stratigraphy, distribution and sourves constraints. Journal of Volcanology and Geothermal Research, 63, 59-87.

MELLAART J., 1967 - Catal Hüyük : a Neolithic city in Anatolia. Thames and Hudson, $201 \mathrm{p}$.

MOURALIS D., KUZUCUOGLU C., PASTRE J.-F., TURKECAN A., 2001 - Le volcanisme récent de Cappadoce : manifestations volcaniques du Pléistocène supérieur et de l'Holocène enregistrées dans le maar d'Eski Acıgöl - Résultats préliminaires. $8^{i m}$ Congrès Français de Sédimentologie, Association des Sédimentologistes Français, Orléans, 12-14 novembre 2001, Livre des résumés, pp. 251-252.

PASTRE J-F, KUZUCUOGLU C FONTUGNE M, KARABIYIKUOGLU M., ERCAN T., TÜRKECAN A., 1998 - Séquences volcanisées et corrélations tephrologiques au NE du Hasan Dag (haut bassin de la Melendiz, Anatolie centrale, Turquie). Quaternaire, 9, 169-183.

OLANCA K., 1994 (non publié) - Géochimie des laves quaternaires de Cappadoce (Turquie). Les appareils monogéniques. Thèse de doctorat, Université Blaise Pascal, Clermont Ferrand II, $156 \mathrm{p}$

SLIMAK L., ROCHE H., 2001 (non publié) - Évaluation archéologique du secteur paléolithique - Kaletepe Deresi 3, rap port de fouille, $8 \mathrm{p}$.

TÜRKECAN A. ACARLAR M., DÖNMER M., HEPSEN N., BILGIN R. 1998 - Kayseri (Bünyan, Develi, Tomarza) yöresinin jeoloji ve volkanik kayaçlarin petrologisi. MTA - Genel Müdürlügü - Jeoloji Etütleri Daiseri Baskanligi, Ankara, v + 208 p. + cartes géologiques au 1:25000 (26 feuilles couvrant l'ensemble du volcan).

WOLDRING H. 1997 - Late Glacial and Holocene vegetation history of central Anatolia : the palynological record of Eski Acıgöl. INQUA Regional Symposium on Late Pleistocene in the Eastern Mediterranean, Ankara, Avril 1997; Livre des résumé.

YILDIRIM T., ÖZGÜR R., 1981 - The caldeira of Acıgöl. Jeomorfoloji Dergisi, 10, 59-70. 